\title{
El análisis de errores en la resolución de ecuaciones diferenciales ordinarias. Una metodología para desarrollar la competencia matemática
}

\section{The analysis of errors in the solution of ordinary differential equations. A methodology to develop mathematical competence}

Juan Jesús Barbarán Sánchez

Departamento de Álgebra, Facultad de Educación y Humanidades de Ceuta, Universidad de Granada barbaran@ugr.es

José Antonio Fernández Bravo

Cátedra Conchita Sánchez de Investigación para la Educación Matemática, Facultad de Educación y Ciencias Sociales, Universidad Camilo José Cela

jafernandez@ucjc.edu

RESUMEN • En este artículo presentamos los resultados de un estudio llevado a cabo con alumnos de segundo de Ingeniería Técnica en Informática de Gestión, en el que observamos si el uso continuado en el aula de una metodología basada en el análisis de errores cuando estudian ecuaciones diferenciales ordinarias mejora el desarrollo de la competencia matemática. El diseño de la investigación fue cuasi experimental, comparativo con un grupo experimental y un grupo control. Los resultados obtenidos muestran que la metodología basada en el análisis de errores es mejor que la metodología tradicional para el desarrollo de la competencia matemática.

PALABRAS CLAVE: ecuaciones diferenciales ordinarias; análisis de errores; competencia matemática.

ABSTRACT $\bullet$ In this paper we present the results of a study carried out with students of $2^{\text {nd }}$ Technical Engineering in Computer Management, which studied if the continued use of a methodology based on error analysis improves their mathematical competence when they study ordinary differential equations. The investigation design was quasi-experimental comparison with an experimental group and a control group. The results show that the methodology based on error analysis is better than traditional methodology to develop mathematical competence.

KEYWORDS: ordinary differential equations; analysis of errors; mathematical competence.

Fecha de recepción: febrero 2013 - Aceptado: mayo 2013 


\section{INTRODUCCIÓN}

Las ecuaciones diferenciales ordinarias (EDO) son una herramienta básica para los profesionales de especialidades relacionadas con la ciencia y la tecnología, como es el caso de los ingenieros informáticos. Esto es debido a que las EDO posibilitan describir fenómenos basados en la variación y, por tanto, permiten modelizar y resolver problemas procedentes de contextos muy diversos.

Artigue (1992) realizó un estudio con el propósito de explorar la enseñanza y aprendizaje de las EDO de primer orden mediante un estudio cualitativo de sus soluciones, Habre (2000) identificó las diferentes estrategias que utilizan los estudiantes al resolver EDO y Rasmussen (2001) desarrolló un marco para interpretar la comprensión de los estudiantes y las dificultades que tienen con ideas matemáticas centrales de las ecuaciones diferenciales. Camacho, Perdomo y Santos Trigo (2007) observaron que, en líneas generales, la idea que tienen los estudiantes de resolver una ecuación diferencial se reduce a la aplicación de algoritmos específicos de clasificación y resolución de las EDO. Rasmussen y Whitehead (2003) realizaron una revisión de distintos trabajos relacionados con la enseñanza y el aprendizaje de las EDO, identificando distintas estrategias, dificultades y formas de comprender que muestran los estudiantes en relación con la creación, interpretación y coordinación de distintos sistemas de representación (incluyendo diagramas de fase y de bifurcación) y la formulación de predicciones justificadas acerca del comportamiento de las funciones solución.

Un modelo de enseñanza propuesto en el ámbito de la investigación en educación matemática es el realizado en el marco del proyecto Inquiry-Oriented Differential Equation (IO-DE), en el que se interpreta el concepto de EDO como una expresión que indica la evolución de una función en el tiempo (Rasmussen y Kwon, 2007). Los estudiantes que participan en este proyecto trabajan en un ambiente en el que se promueve la discusión, el planteamiento de conjeturas, la justificación de las ideas y la creación de métodos de resolución propios (Rasmussen y Blumenfeld, 2007). Distintas investigaciones han mostrado que estos estudiantes obtienen mejores resultados que otros grupos que no participaron en este proyecto, sobre todo en actividades relacionadas con la modelización y el análisis del comportamiento de las soluciones de una ecuación (Rasmussen, Kwon, Allen, Marrongelle y Burtch, 2006), además de tener una mayor capacidad de retención de sus conocimientos y habilidades matemáticas (Kwon, Rasmussen y Allen, 2005).

Es un hecho que nuestros alumnos cometen errores en sus producciones cuando trabajan cualquier dominio matemático. Analizar esos errores es una fuente valiosa de información, como ya consideró Weiner (1922) (citado por Radatz [1980]), quien inició la investigación didáctica orientada al estudio de errores. Existen numerosas investigaciones en las que se clasifican y categorizan los errores usando diferentes enfoques (Davis, 1984; Booth, 1984; Esteley-Villarreal, 1996; Azcárate et al., 1996; Rico, 1998; Astolfi, 1999). Socas (1997) considera tres ejes, no disjuntos, que nos permiten analizar el origen del error. De esta forma, podemos situar los errores que cometen los alumnos en relación con tres orígenes distintos: obstáculo, ausencia de sentido y actitudes afectivas y emocionales. Entre las dificultades que existen en el proceso de enseñanza-aprendizaje de las ecuaciones diferenciales se han encontrado obstáculos en la integración de distintos registros de representación (Hernández, 1995).

El desarrollo adecuado de la competencia matemática de nuestros alumnos de los primeros cursos de Ingeniería es básico para que puedan avanzar en otros conocimientos propios de su disciplina. En 1999 se inició el proyecto Tuning, que pretende potenciar el Espacio Europeo de Educación Superior, en el que se trata de uniformar los programas de formación de algunas disciplinas entre las que se encuentra las Matemáticas por medio de, entre otros aspectos, competencias genéricas y específicas (González y Wagenaar, 2003: 129-130). Entre las capacidades genéricas que se espera que desarrollen los estudiantes aparecen la elaboración y el sostenimiento de argumentos, la resolución de problemas, las habilidades comunicativas y la capacidad de análisis y de síntesis. Además, se incluyen las siguientes 
capacidades específicas: la de idear una demostración, la de construir un modelo matemático a partir de una situación dada y la de resolver problemas utilizando herramientas matemáticas. Las capacidades descritas están íntimamente ligadas con las propuestas por Niss (2002), como veremos más adelante. Este proyecto considera las EDO como contenido de nivel 2 dentro de las competencias clave específicas del área de Matemáticas para un estudiante universitario. A partir del proyecto Tuning surgieron dos proyectos latinoamericanos que enfatizan las competencias en la educación universitaria: el Tuning América Latina y el 6x4 UEALC.

En este artículo utilizamos el análisis de errores como propuesta metodológica. Cuando se discute por qué algo no es correcto, el sujeto debe mantener la atención en los argumentos esgrimidos por sus interlocutores y aportar los suyos, para lo que es necesario que piense por sí mismo. El análisis de errores es uno de los instrumentos de explicación más poderosos. Sin embargo, a pesar de su potencialidad, es un instrumento que no se usa de forma sistemática en el aula. Esto sugiere la oportunidad de extender su utilización, adaptándola también a las variadas situaciones, cursos y niveles de instrucción (Mammino, 2002: 168).

Es importante estudiar estrategias innovadoras que desarrollen la competencia matemática en los procesos de aprendizaje de las EDO de nuestros alumnos. Nuestra investigación viene a complementar investigaciones previas sobre la enseñanza y aprendizaje de las EDO (Guerrero, Camacho y Mejía, 2010) y sobre el empleo del análisis de errores para aclarar conceptos (Love y Mammino, 1997; Mammino, 2002). Nuestro objetivo consiste en estudiar si existe relación entre la aplicación continuada en un aula con alumnos de segundo de Ingeniería Técnica de Informática de Gestión de una metodología basada en el análisis de errores y el desarrollo de su competencia matemática. Consideramos que los resultados de este estudio permitirán considerar el análisis de errores como una técnica pedagógica válida, probada empíricamente en el aula, en procesos de enseñanza y aprendizaje de las EDO.

\section{LA COMPETENCIA MATEMÁTICA EN EL ESTUDIO DE EDO}

Las ecuaciones diferenciales sirven como modelo matemático para estudiar problemas que surgen en disciplinas muy diversas (física, química, biología, economía, etc.). Algunos ejemplos son el modelo malthusiano de crecimiento de la población de un país, el modelo de crecimiento de una célula o el modelo de determinación de la edad de una pintura o de un fósil.

Uno de los orígenes de las ecuaciones diferenciales lo encontramos en los intentos de resolver problemas de la física como, por ejemplo, el problema de la braquistócrona ${ }^{1}$ mediante el cálculo diferencial a finales del siglo XVII.

En este estudio, entendemos por competencia matemática la capacidad de argumentar matemáticamente, modelizar matemáticamente y utilizar símbolos y formalismos matemáticos con las ecuaciones diferenciales.

La noción de competencia matemática se ha usado con distintas interpretaciones (Schwartz, 1995; Abrantes, 2001; Niss, 2002; Schoenfeld, 2007; Rico y Lupiáńez, 2008; Britta, 2010; Kuntze, 2010). Mogens Niss, matemático danés, es uno de los investigadores que más ha contribuido a crear un marco teórico acerca de la competencia matemática. Introdujo en 1999 ocho competencias matemáticas específicas. El primer grupo de competencias tiene que ver con la habilidad para preguntar y responder cuestiones en matemáticas y por medio de las matemáticas, y son las siguientes: pensar matemáticamente, modelizar matemáticamente, plantear y resolver problemas matemáticos y argumentar mate-

1. Considérese una partícula que se traslada, dentro de un campo de fuerzas constante, desde un punto $\left(\mathrm{x}_{1}, \mathrm{y}_{1}\right)$ donde inicialmente se halla en reposo hasta otro punto $\left(\mathrm{x}_{2}, \mathrm{y}_{2}\right)$ más abajo que el primero. El problema consiste en determinar la trayectoria que permita a la partícula trasladarse de uno a otro punto en el menor tiempo posible (Marion, 1998: 208). 
máticamente. El segundo grupo de competencias se refiere a la habilidad de utilizar el lenguaje y las herramientas matemáticas, y lo componen: representar entidades matemáticas (objetos y situaciones), utilizar símbolos y formalismos matemáticos, comunicarse en, con y sobre las matemáticas y utilizar recursos y herramientas.

Introducimos en la tabla I la relación existente entre las competencias genéricas que se definen en el proyecto Tuning (González y Wagenaar, 2003: 129-130) y ciertas competencias matemáticas específicas propuestas por Niss (2002).

Tabla I.

Relación entre las competencias genéricas del proyecto Turing

(González y Wagenaar, 2003) y las de Niss (2002)

\begin{tabular}{|l|l|}
\hline \multicolumn{1}{|c|}{ Competencias genéricas del proyecto Tuning } & \multicolumn{1}{c|}{ Competencias matemáticas especificas de Niss } \\
\hline Elaboración y sostenimiento de argumentos & Argumentar matemáticamente \\
\hline Resolución de problemas & Plantear y resolver problemas matemáticos \\
\hline Habilidades comunicativas & Comunicarse en, con y sobre las matemáticas \\
\hline Capacidad de análisis y de síntesis & Pensar matemáticamente \\
\hline
\end{tabular}

Si nos centramos en las competencias específicas propuestas en el proyecto Tuning, observamos que también existe un paralelismo con ciertas competencias presentadas por Niss (2002), aunque estas tienen un espectro descriptivo más amplio y variado.

Tabla II.

Relación entre las competencias específicas del proyecto Turing

(González y Wagenaar, 2003) y las de Niss (2002)

\begin{tabular}{|l|l|}
\hline \multicolumn{1}{|c|}{ Competencias especificas del proyecto Tuning } & \multicolumn{1}{|c|}{ Competencias matemáticas especificas de Niss } \\
\hline Capacidad de idear una demostración & Argumentar matemáticamente \\
\hline Capacidad de construir un modelo matemático & Modelizar matemáticamente \\
\hline $\begin{array}{l}\text { Capacidad para resolver problemas utilizando herramientas } \\
\text { matemáticas }\end{array}$ & Plantear y resolver problemas matemáticos \\
\hline
\end{tabular}

Consideramos que el enfoque llevado a cabo por Niss (2002) se adapta más al enfoque que queremos darle a este estudio por su nivel de concreción en lo que se refiere a los descriptores de las competencias matemáticas específicas.

En nuestra investigación, la competencia matemática la vamos a describir a través de las siguientes competencias matemáticas específicas: argumentar matemáticamente, modelizar matemáticamente y utilizar símbolos y formalismos matemáticos. Una descripción de las capacidades evaluadas en este estudio son las que aparecen en la tabla III. 
Tabla III.

Capacidades evaluadas en este estudio (adaptadas de Niss [2002])

\begin{tabular}{|l|l|}
\hline \multicolumn{1}{|c|}{ Competencias matemáticas especificas } & \multicolumn{1}{c|}{ Capacidades } \\
\hline Argumentar matemáticamente & $\begin{array}{l}\text { - Diseñar argumentos matemáticos formales e informales y transfor- } \\
\text { mar los argumentos heurísticos en demostraciones válidas. }\end{array}$ \\
\hline Modelizar matemáticamente & - Analizar los fundamentos y propiedades de modelos existentes. \\
\hline Utilizar símbolos y formalismos matemáticos & $\begin{array}{l}\text { - Entender la naturaleza y las reglas de los sistemas matemáticos (sin- } \\
\text { taxis y semántica). } \\
\text { - Trabajar con enunciados y expresiones que contengan símbolos y } \\
\text { fórmulas. }\end{array}$ \\
\hline
\end{tabular}

Otro aspecto de vital importancia en esta investigación es la metodología basada en el análisis de errores. Se trata de un instrumento pedagógico muy valioso ya que discutir por qué algo no es correcto atrae la atención de los alumnos y los hace pensar por sí mismos (Mammino, 2002). Sin embargo, a pesar del importante potencial que posee, es un instrumento que no se utiliza habitualmente en el aula. Tomamos como referente a Mammino (2002) para la descripción de la metodología basada en el análisis de errores.

\section{PLANTEAMIENTO DEL ESTUDIO}

El objetivo principal de este estudio es analizar las consecuencias que tiene el uso de una metodología basada en el análisis de errores en el desarrollo de la competencia matemática de alumnos de segundo de Ingeniería Técnica en Informática de Gestión cuando estudian EDO.

El diseño de la investigación llevada a cabo fue cuasi experimental, comparativo de dos grupos. Se eligieron al azar 9 alumnos que conformaron el grupo experimental y otros 9 que formaron el grupo control. Como describiremos más adelante, los alumnos fueron evaluados antes y después de la aplicación de la metodología basada en el análisis de errores, lo que subraya en el diseño la característica pretest-postest.

La investigación se realizó en el ámbito de la Ingeniería Técnica en Informática de Gestión, titulación que oferta la Universidad de Granada en el campus de Ceuta. Los participantes fueron 18 alumnos, de entre 19 y 23 años de edad, que cursaban la asignatura Cálculo numérico. En la muestra había una mujer; el resto eran hombres.

Las variables utilizadas en nuestro estudio son las siguientes:

A. Variable independiente: la metodología basada en el análisis de errores.

B. Variables dependientes: argumentar matemáticamente, modelizar matemáticamente y utilizar símbolos y formalismos matemáticos. Se trata de tres variables cuantitativas cuyo rango de valores aparece en la tabla IV, así como el ejercicio que se usó para evaluarlas.

Tabla IV.

Descripción de las variables dependientes

\begin{tabular}{|l|c|c|}
\hline \multicolumn{1}{|c|}{ Variable } & Rango de valores & Ejercicio \\
\hline Argumentar matemáticamente & $0-40$ & 2 \\
\hline Modelizar matemáticamente & $0-20$ & 3 \\
\hline Utilizar símbolos y formalismos matemáticos & $0-40$ & 1 \\
\hline
\end{tabular}


Para evaluar las variables dependientes se diseñó un instrumento que aparece como anexo II, que fue validado por el método de triangulación de expertos y experimentado con éxito, antes de que se iniciase este estudio, con 36 estudiantes de segundo de Ingeniería Técnica en Informática de Gestión que no participaron en esta experiencia.

C. Variable interviniente: el nivel de conocimiento matemático previo. A nuestro juicio, esta variable podía estar sistemáticamente relacionada con la variable independiente, y podía afectar de forma diferencial a los valores de las variables dependientes. Con el fin de medir la variable interviniente se diseñó una prueba de nivel de conocimiento matemático que figura como anexo I. Este instrumento fue validado siguiendo el mismo procedimiento que el instrumento citado en el apartado B.

Para los alumnos del grupo experimental, se confeccionó un cuaderno de trabajo en el que aparecían un total de 32 ejercicios resueltos de EDO que contenían distintos tipos de errores, siguiendo la clasificación realizada por Movshovitz-Hadar, Zaslavsky e Inbar (1987) y citados por Rico (1998):

- Datos mal utilizados. Se incluyen aquí aquellos errores que se han producido por alguna discrepancia entre los datos que aparecen en una cuestión y el tratamiento que le ha dado el alumno.

- Interpretación incorrecta del lenguaje. Se incluyen en este caso los errores debidos a una traducción incorrecta de hechos matemáticos descritos en un lenguaje simbólico a otro lenguaje simbólico distinto.

- Inferencias no válidas lógicamente. Esta categoría incluye aquellos errores que se producen por falacias de razonamiento y no se deben al contenido específico.

- Teoremas o definiciones deformados. Se incluyen aquí aquellos errores que se producen por deformación de un principio, regla o definición identificable.

- Falta de verificación de la solución. Se incluyen aquí los errores que se presentan cuando cada paso en la realización de la tarea es correcto, pero el resultado final no es la solución de la pregunta planteada; si el solucionador hubiese contrastado la solución con el enunciado, el error habría podido evitarse.

- Errores técnicos. Se incluyen en esta categoría los errores de cálculo, errores al tomar datos de una tabla, errores en la manipulación de símbolos algebraicos y otros derivados de la ejecución de algoritmos básicos.

A estos errores le sumamos un tipo considerado por los autores de este artículo:

- Error de linealización del producto (cociente) de la integral. Consiste en que el alumno calcula la integral de un producto (cociente) como el producto (cociente) de las integrales.

Había ejercicios en los que se incluyó más de un error.

Para los alumnos del grupo control, se elaboró una relación de ejercicios que contenía los enunciados de los mismos ejercicios que el cuaderno de trabajo antes citado pero sin soluciones.

Para la recogida de datos se utilizaron:

a) Prueba de nivel de conocimiento matemático: la prueba consta de un total de 5 preguntas que versan sobre resolución de ecuaciones, conjuntos numéricos y cálculo de límites, derivadas e integrales en un tiempo de 1 hora. Puede consultarse en el anexo I.

b) Prueba de EDO: la prueba, que figura en el anexo II, está formada por 3 ejercicios; en el primero se le pide al alumno que identifique y resuelva $4 \mathrm{EDO}$, en el segundo se le plantean dos cuestiones relativas al concepto de solución de una EDO y en el tercero se le proporciona una EDO con condiciones iniciales que modeliza un fenómeno y se le pide que utilice su conoci- 
miento sobre EDO para resolver dos cuestiones relativas al modelo descrito. El tiempo de que dispusieron para su resolución fue de 2 horas. Las variables "argumentar matemáticamente», «modelizar matemáticamente» y «utilizar símbolos y formalismos matemáticos» se midieron mediante la puntuación obtenida en los ejercicios 2,3 y 1 , respectivamente.

En esta investigación se han considerado como datos las respuestas de los alumnos a las dos pruebas que acabamos de describir.

El estudio lo podemos dividir en tres fases:

- Fase pretest. En esta etapa, que tuvo lugar a principios de marzo de 2010, se les administró a todos los alumnos de la muestra, de forma simultánea y en la misma aula, la prueba de nivel de conocimiento matemático que figura como anexo I para determinar su nivel de conocimiento matemático.

- Fase de intervención. Se llevó a cabo mediante la aplicación de la metodología basada en el análisis de errores a los alumnos del grupo experimental en 26 sesiones a lo largo de 4 meses. Todas las sesiones tuvieron 1 hora de duración, salvo la dedicada a la prueba de conocimiento de EDO, que duró 2 horas. El horario fijado para estas sesiones coincidió en los dos grupos. Los contenidos explicados a ambos grupos fueron: la definición de EDO, la resolución de EDO de primer orden (variables separadas, homogéneas, Bernouilli, diferencial exacta) y con condiciones iniciales (junto a problemas modelizados por este tipo de herramientas matemáticas). La distribución temporal de las sesiones aparece en la tabla $\mathrm{V}$.

Tabla V.

Distribución temporal de las sesiones

\begin{tabular}{|l|c|}
\hline \multicolumn{1}{|c|}{ Actuación-contenidos tratados } & $N .^{\text {o de sesión }}$ \\
\hline Prueba inicial de conocimientos matemáticos & 1 \\
\hline Definición de EDO & $2-3$ \\
\hline Resolución de EDO de primer orden & $4-15$ \\
\hline $\begin{array}{l}\text { Resolución de EDO de primer orden con condiciones ini- } \\
\text { ciales y problemas }\end{array}$ & $16-25$ \\
\hline Prueba de EDO & 26 \\
\hline
\end{tabular}

Los ejercicios que se utilizaron con los grupos experimental y control fueron los mismos, con la única salvedad de que los que se trabajaron en el grupo experimental ya aparecían resueltos con errores de los descritos anteriormente, mientras que con el grupo control se siguió una metodología tradicional en la que el profesor resolvió en la pizarra los ejercicios sin usar el análisis de errores. Los ejercicios se propusieron en el mismo orden en ambos grupos y entremezclados con las explicaciones teóricas. En la búsqueda de errores con los estudiantes del grupo experimental se siguieron las siguientes etapas basadas en las descritas por Fernández-Bravo (2010):

- Apertura: se le planteó al alumno la actividad insistiéndole en que debía descubrir el error o errores que aparecen en el ejercicio resuelto. Seguidamente nos aseguramos de que había comprendido perfectamente lo que había que hacer.

- Ejecución: fase en la que se realizó la propuesta. La ejecución pudo realizarse en gran grupo o grupo-clase, a partir de un diálogo en común; en parejas de alumnos, o de forma individual.

- Contrastación: fase en la que se contrastaron las ideas mediante el diálogo. Si la fase anterior se había realizado de forma individual, esta fase se llevó a cabo por parejas. Si la ejecución se 
llevó a cabo por parejas, esta fase se realizó por parejas de parejas. Si en la fase anterior había intervenido el grupo-clase, la fase en la que estamos ahora formó parte de la anterior.

- Exposición: fase en la que intervino el grupo-clase con la libre participación de todos y cada uno de los alumnos que quisieron exponer sus ideas. Mediante el diálogo en gran grupo y las preguntas del profesor, se canalizaron las ideas y se recogieron las estrategias matemáticas que se habían reconocido como inválidas.

- Finalización: se escribieron y anotaron las conclusiones que se obtuvieron: conceptuales, procedimentales, etc.

- Fase postest. Esta etapa se desarrolló durante el mes de junio de 2010. A los alumnos se les aplicó el mismo día, a la misma hora y en la misma aula la prueba de EDO que figura como anexo II.

\section{RESULTADOS Y DISCUSIÓN DEL ESTUDIO}

En primer lugar, y como aparece en la tabla VI, se estudió mediante un análisis de la varianza si existían diferencias estadísticamente significativas $(\mathrm{p}<0,05)$ entre el grupo experimental y control en lo que se refiere a la variable interviniente «nivel de conocimiento matemático previo». La respuesta fue negativa. Por tanto, esta variable no ha influido en los resultados del estudio.

Tabla VI.

Resultados del estudio estadístico

\begin{tabular}{|l|c|c|}
\hline & F & Sig. (bilateral) \\
\hline Nivel de conocimiento matemático previo & 0,893 & 0,424 \\
\hline
\end{tabular}

Para verificar los cambios pretest-postest en las variables dependientes consideradas (argumentar matemáticamente, modelizar matemáticamente y utilizar símbolos y formalismos matemáticos), respecto a la utilización o no de la metodología basada en el análisis de errores (variable independiente), se comprobó la normalidad de las muestras, y se utilizó la prueba T de Student junto con la prueba de Levene para el contraste de las hipótesis estadísticas. Se analizó el estadístico F y su significación (Sig. F) para los dos grupos. El contraste de hipótesis estadísticas lo basamos en un contraste de igualdad de medias de dos poblaciones normales de varianzas desconocidas. El contraste fue bilateral, considerando como hipótesis las siguientes, por ejemplo, para la variable «argumentar matemáticamente»:

- Hipótesis nula: no existirán diferencias estadísticamente significativas entre los resultados obtenidos por el grupo experimental y control en la fase postest, para la variable «argumentar matemáticamente».

- Hipótesis alternativa: existirán diferencias estadísticamente significativas entre los resultados obtenidos por el grupo experimental y control en la fase postest, para la variable «argumentar matemáticamente».

De la misma forma se llevaron a cabo contrastes de hipótesis para las variables «modelizar matemáticamente» $\mathrm{y}$ «utilizar símbolos y formalismos matemáticos».

La existencia de diferencias significativas nos haría rechazar la hipótesis nula y aceptar la hipótesis alternativa. Los resultados del estudio estadístico realizado en la fase postest, que aparecen en la tabla VII, nos permiten deducir que existen diferencias estadísticamente significativas entre el grupo experimental y control en las tres variables dependientes consideradas. 
Tabla VII.

Resultados del estudio estadístico

\begin{tabular}{|l|c|c|}
\hline & F & Sig. (bilateral) \\
\hline Utilizar símbolos y formalismos matemáticos & 9,352 & 0,003 \\
\hline Argumentar matemáticamente & 10,404 & 0,000 \\
\hline Modelizar matemáticamente & 6,083 & 0,002 \\
\hline
\end{tabular}

Todos los análisis fueron realizados con el programa Statistical Package for the Social Sciences (SPSS), versión 15.0 .

\section{CONSIDERACIONES FINALES}

El estudio presentado en este artículo ha pretendido comprobar, a través de la investigación realizada, que el uso continuado en el aula de una metodología basada en el análisis de errores con alumnos de Ingeniería Técnica en Informática de Gestión mejora su competencia matemática cuando estudian EDO. Esta afirmación se apoya en el desarrollo de las siguientes competencias matemáticas específicas: argumentar matemáticamente, modelizar matemáticamente y utilizar símbolos y formalismos matemáticos.

En la mayor parte de los casos las concepciones erróneas tienen su origen en cursos precedentes y su corrección no es tarea fácil, como afirma Mammino (2002). Con esta investigación ponemos de manifiesto que la metodología en la que el análisis de errores sea el elemento principal hace que los alumnos asimilen mejor los conceptos relacionados con las EDO de primer orden y desarrollen su competencia matemática.

En el futuro sería conveniente confirmar estadísticamente estos resultados ampliando la muestra con alumnos de otras titulaciones.

Para mejorar la enseñanza y el aprendizaje de las ecuaciones diferenciales en los primeros cursos universitarios de ciencia e ingeniería, Guerrero, Camacho y Mejía (2010) plantearon como línea de trabajo futura la búsqueda de estrategias de actuación. Como respuesta a la cuestión formulada por estos autores, planteamos el uso de una metodología basada en el análisis de errores.

\section{REFERENCIAS BIBLIOGRÁFICAS}

Abrantes, P. (2001). Mathematical competence for all: Options, implications and obstacles. Educational Studies in Mathematics, 47, pp. 125-143.

http://dx.doi.org/10.1023/A:1014589220323

Artigue, M. (1992). Functions from an Algebraic and Graphic Point of View: Cognitive Difficulties and Teaching Practices. En G. Harel y E. Dubinsky (eds.). The concept of function. Aspects of epistemology and Pedagogy, 25. MAA notes USA, pp. 109-132.

Astolfi, J.P. (1999). El «erron», un medio para enseñar. Sevilla: Díada Editora.

Azcárate, C.; Bosch, D.; Casadevall, M. y Casellas, E. (1996). Cálculo diferencial e integral. España: Editorial Síntesis.

Bоотн, L.R. (1984). Algebra: children's strategies and errors. Windsor: NFER-Nelson.

BRItTA, C. (2010). Heureka-ich hab's. Stationsarbeit zur Problemlösekompetenz. Mathematik 5 bis 10, 13, pp. 26-29. 
Camacho, M.; Perdomo, J. y Santos Trigo, M. (2007). La resolución de problemas en los que interviene el concepto de Ecuación Diferencial Ordinaria: Un estudio exploratorio. En M. Camacho, P. Bolea, P. Flores, B. Gómez, J. Murillo y M. ${ }^{a}$ González (eds.). Investigación en Educación Matemática. Comunicaciones de los grupos de investigación. Tenerife: SEIEM, pp. 87-106.

Davis, R. (1984). Learning Mathematics. The cognitive Science Approach to Mathematics Education. Australia: Croom Helm. http://dx.doi.org/10.1016/0023-9690(84)90004-3

Esteley, C. y Villareal, M. (1996). Análisis y categorización de errores en Matemática. Revista de Educación Matemática, 11(1). Universidad Nacional de Córdoba: Córdoba, pp. 16-35.

Fernández-Bravo, J.A. (2010). La resolución de problemas matemáticos. Creatividad y razonamiento en la mente de los niños. Madrid: Grupo Mayéutica.

González, J. y WagenaAR, R. (eds.) (2003). Tuning Educational Structures in Europe. Informe Final. Fase 1. Bilbao, España: Universidad de Deusto.

Guerrero, C.; Camacho, M. y Mejía, H.R. (2010). Dificultades de los estudiantes en la interpretación de las soluciones de ecuaciones diferenciales ordinarias que modelan un problema. Enseñanza de las Ciencias, 28 (3), pp. 341-352.

Habre, S. (2000). Exploring students'strategies to solve ordinary differential equations in a reformed setting. Journal of Mathematical Behavior, 18 (4), pp. 455-472. http://dx.doi.org/10.1016/S0732-3123(00)00024-9

Hernández, A. (1995). Obstáculos en la Articulación de los Marcos Numérico, Gráfico y Algebraico en relación con las Ecuaciones Diferenciales. Tesis doctoral. México: Cinvestav.

Kuntze, S. (2010). Zur Beschreibung von Kompetenzen des mathematischen Modellierens konkretisiert an inhaltlichen Leitideen: Eine Diskussion von Kompetenzmodellen als Grundlage für Förderkonzepte zum Modellieren im Mathematikunterricht. Mathematikunterricht (Seelze), 56(4), pp. 4-19.

Kwon, O.; Rasmussen, C. y Allen, K. (2005). Students' Retention of Mathematical Knowledge and Skills in Differential Equations. School Science and Mathematics, 105(5), pp. 1-13. http://dx.doi.org/10.1111/j.1949-8594.2005.tb18163.x

Love, A. y Mammino, L. (1997). Using the analysis of errors to improve students' expression in the sciences. Zimbabwe Journal of educational Research, 9(1), pp. 1-17.

Mammino, L. (2002). Empleo del análisis de errores para aclarar conceptos de química general. Enseñanza de las Ciencias, 20 (1), pp. 167-173.

Marion, J.B. (1998). Dinámica clásica de las partículas y sistemas. Barcelona: Reverté.

Movshovitz-Hadar, N.; Zaslavsky, O. y Inbar, S. (1987). An empirical classification model for errors in High School Mathematics. Journal for Research in Mathematics Education, 18, pp. 3-14. http://dx.doi.org/10.2307/749532

Niss, M. (2002). Mathematical competencies and the learning of mathematics: The Danish kom project. Disponible en: http://www7.nationalacademies.org/mseb/mathematical_competencies_and_the_ learning_of_mathematics.pdf. (Última consulta, noviembre del 2012).

Radatz, H. (1980). Student's errors in the mathematical learning process: a survey. For the learning of Mathematics, 1 (1), pp. 16-20.

Rasmussen, C. (2001). New directions in differential equations: A framework for interpreting students' understandings and difficulties. Journal of Mathematical Behavior, 20 (1), pp. 55-87. http://dx.doi.org/10.1016/S0732-3123(01)00062-1 
Rasmussen, C. y Blumenfeld, H. (2007). Reinventing solutions to systems of linear differential equations: A case of emergent models involving analytic expressions. Journal of Mathematical Behavior, 26, pp. 195-210. http://dx.doi.org/10.1016/j.jmathb.2007.09.004

Rasmussen, C. y Kwon, O. (2007). An inquiri-oriented approach to undergraduate mathematics. Journal of Mathematical Behavior, 26, pp. 189-194. http://dx.doi.org/10.1016/j.jmathb.2007.10.001

Rasmussen, C.; Kwon, O.; Allen, K.; Marrongelle, K. y Burtch, M. (2006). Capitalizing on Advances in Mathematics and K-12 Mathematics Education in Undergraduate Mathematics: An Inquiry-Oriented Approach to Differential Equations. Asia Pacific Education Review, 7 (1), pp. 85-93.

http://dx.doi.org/10.1007/BF03036787

Rasmussen, C. y Whitehead, K. (2003). Learning and teaching ordinary differential equations. En A. Selden y J. Selden (eds.). MAA Online Research Sampler.

Rico, L. (1998). Errores en el aprendizaje de las Matemáticas. En J. Kilpatrick, L. Rico y P. Gómez (eds.). Educación matemática. México: Grupo Editorial Iberoamérica.

Rico, L. y Lupiáñez, J.L. (2008). Competencias matemáticas desde una perspectiva curricular. Madrid: España: Alianza Editorial.

Schoenfeld, A. (2007). Assessing Mathematical Proficiency. Cambridge: University Press. http://dx.doi.org/10.1017/CBO9780511755378

Schwartz, R. (1995). Is Mathematical Competence Innate? Philosophy of Science, 62(2), pp. 227-240. http://dx.doi.org/10.1086/289854

Socas, M. (1997). Dificultades, obstáculos y errores en el aprendizaje de las matemáticas en la Educación Secundaria. En L. Rico. La Educación Matemática en la Enseñanza Secundaria. Barcelona: ICE Universitat de Barcelona/HORSORI, pp. 125-154. 


\section{ANEXO I \\ (Prueba de nivel de conocimiento matemático)}

1.

i) Define el valor absoluto de un número real.

ii) Resuelve la ecuación $|x-3|=2$

2.

i) De entre los siguientes números: $0 . \widehat{6}, 0.666, \sqrt{5}$, ¿cuáles son racionales?

ii) ¿Cuál es la relación entre 0.9 y 1 ?

3. Calcula los siguientes límites:
i) $\lim _{x \rightarrow-2} x^{3}-2 x^{2}-x+5$
ii) $\lim _{x \rightarrow 0} \frac{x^{3}+5 x}{6 x}$
iii) $\lim _{x \rightarrow+\infty} \frac{e^{x+1}}{\ln \left(x^{2}+1\right)}$

donde ln denota el logaritmo neperiano.

4. Calcula las derivadas de las siguientes funciones:
i) $f(x)=e^{x^{3}-4 x^{2}+x-1}$
ii) $f(x)=\ln \left(3 x^{2}-x+2\right)$
iii) $\mathrm{f}(\mathrm{x})=\sin (\cos (\mathrm{x}))$

5. Calcula:
i) $\int x \ln (x) d x$
ii) $\int_{-1}^{1} \frac{1}{\mathrm{x}^{2}} \mathrm{dx}$ 


\section{ANEXO II \\ (Prueba de EDO)}

1. Resuelve las siguientes ecuaciones diferenciales:
i) $y^{\prime}=e^{x}-\frac{3 x}{x^{2}-1}$
ii) $\left(3 t^{2} x+x^{3}\right) x^{\prime}+2 t^{3}=0$
iii) $x y^{\prime}+y=y^{2} \log (x)$
iv) $t \cos (t+x)+\operatorname{sen}(t+x)+t \cos (t+x) x^{\prime}=0$

2. Responde razonadamente a las siguientes cuestiones:

i) ¿Puede ser la función $\mathrm{f}(\mathrm{t})=\mathrm{t}^{2}$, con $t \in \mathbb{R}$, solución de una ecuación diferencial lineal de primer orden homogénea? ¿Y de una ecuación lineal de primer orden no homogénea?

ii) ¿Pueden ser $\mathrm{f}(\mathrm{t})=\mathrm{e}^{\mathrm{t}} \mathrm{y} \mathrm{g}(\mathrm{t})=\mathrm{e}^{-\mathrm{t}}$, con $t \in \mathrm{R}$, soluciones de una misma ecuación lineal de primer orden homogénea? ¿Y de una ecuación lineal de primer orden no homogénea?

3. La población $\mathrm{P}(\mathrm{t})$ de un suburbio de una gran ciudad en un instante cualquiera se rige por:

$$
\left\{\begin{array}{c}
\frac{d P}{d t}=P\left(10^{-1}-10^{-7} P\right) \\
P(0)=5000
\end{array}\right.
$$

en donde $九$ se mide en meses.

i) ¿Cuál es el valor límite de la población?

ii) ¿En qué momento será la población igual a la mitad de su valor límite? 


\title{
The analysis of errors in the solution of ordinary differential equations. A methodology to develop mathematical competence
}

\author{
Juan Jesús Barbarán Sánchez \\ Departamento de Álgebra, Facultad de Educación y Humanidades de Ceuta, Universidad de Granada \\ barbaran@ugr.es \\ José Antonio Fernández Bravo \\ Cátedra Conchita Sánchez de Investigación para la Educación Matemática, Facultad de Educación y Ciencias Sociales, \\ Universidad Camilo José Cela \\ jafernandez@ucjc.edu
}

The main objective of this study is to analyze the consequences of using a methodology based on the analysis of errors in a classroom with $2^{\text {nd }}$-year students of Technical Engineering in Computer Management in the development of their mathematical competence. Our paper complements previous investigations about teaching and learning Ordinary Differential Equations (ODE) and about the use of the analysis of errors to clarify concepts.

We define mathematical competence as the ability to reason mathematically, model mathematically and handle mathematical symbols and formalisms with ODE.

Participants in this study were 18 students, aged between 19 and 23 years old, who attended the Numerical Analysis course. The investigation design was quasi-experimental comparison with an experimental group and a control group. 9 students were chosen at random to form the experimental group and the other 9 students formed the control group. Students were evaluated before and after the application of the methodology based on the analysis of errors. This fact underlines the characteristic pretest-posttest in the design. A work notebook which contained 32 solved activities containing different types of errors was developed for the students of the experimental group. Among these errors, the following is highlighted by the authors:

- Product (quotient) linearization error that consists of calculating the integral of a product (quotient) of a function as the product (quotient) of the integrals.

A work notebook which contained only the wording of the same 32 activities aforementioned was developed for the students of the control group.

The application of the methodology based on the analysis of errors in the experimental group took place during 26 sessions of 1 hour each, over a total of four months. The schedule established for these sessions coincided in both groups. The contents explained to both groups were the same. Once contents were explained to the students belonging to the experimental group, several solved activities containing different types of errors were presented: errors when operating algebraically, logic errors, technical errors, misinterpreted data, incorrect use of language, etc., and students had to reasonably detect the error. While students in the experimental group were carrying out these activities, students in the control group solved ODE exercises following the traditional methodology and did not analyze errors. Exercises were proposed in the same order in both groups and interspersed with theoretical explanations.

An analysis of variance was used to assess if there were statistically significant differences $(\mathrm{p}<0.05)$ between the experimental group and the control group for the level of mathematical knowledge, and we obtained a negative answer. The results of the statistical study on the posttest phase allows us to deduce that there are statistically significant differences $(\mathrm{p}<0.05)$ between the experimental group and the control group for reasoning mathematically, modeling mathematically and handling mathematical symbols and formalisms with ODE.

With this study we show that when the methodology based on the analysis of errors is the main element considered in teaching ODE, students assimilate concepts and develop their mathematical competence better. We propose the use of a methodology based on the analysis of errors in order to improve the teaching and learning of ODE in the first courses of Science and Engineering studies.

In the future, it would be interesting to confirm these results statistically by expanding the sample with students of other courses. 